\title{
Optical and Radio Monitoring of a Sample of Late-Type Variables
}

\author{
V. F. Esipov ${ }^{1}$, E. E. Lekht ${ }^{1,2}$, M. I. Pashchenko ${ }^{1}$, G. M. Rudnitskij ${ }^{1}$
}

\begin{abstract}
Results of regular observations of about 20 Miras and semiregular variables ( $o$ Cet, $\mathrm{R} \mathrm{Leo,} \mathrm{R} \mathrm{Aql,} \mathrm{U} \mathrm{Her,} \chi$ Cyg, $\mathrm{R}$ Tri, and others) in 1994-1999 are presented. The monitoring includes optical spectroscopy, optical $(U B V)$ and infrared ( $J H K L M)$ photometry, high-resolution radio spectroscopy in the 1.35 -cm water-vapour maser line (on the $22-\mathrm{m}$ radio telescope in Pushchino). Optical spectroscopy shows that the appearance of strong Balmer emission lines in Miras may be a rare event, and the presumed shock waves are thus weak. The $\mathrm{H}_{2} \mathrm{O}$ maser flux generally correlates with visual light curves of the stars with some delay $\Delta \varphi=0.1-0.4 P$ ( $P$ is the variability period). For $\mathrm{R}$ Leo, $\mathrm{R}$ Cas, and $\mathrm{U}$ Aur strong flares in the $\mathrm{H} \alpha$ line, observed in 1997-1998, were followed by corresponding outbursts of the $\mathrm{H}_{2} \mathrm{O}$ maser emission about $2 P$ later. This sequence of events is probably caused by a shock wave propagating outward in the stellar atmosphere and consecutively initiating the $\mathrm{H} \alpha$ emission and, later, the maser flare at a larger distance from the stellar surface.
\end{abstract}

The purpose of this survey is to study spectral variability of a representative sample of Mira-type and semiregular variable stars. Correlation between variability in several wavelength ranges could be a useful tool to study the processes in the inner parts of the circumstellar envelopes, connected with propagation of shock waves.

The optical spectroscopy of the stars was performed in 1994-1999 on the 125-cm ZTE telescope of the Crimean Laboratory of the Sternberg Astronomical Institute. The photometry of the stars was carried out on the $60-\mathrm{cm}$ Zeiss-600 telescope. The early conclusions of Joy (1954) and Gillet, Maurice, \& Baade (1983) about the regular appearance of emission lines in the spectra of these variables in each light cycle near minimum light are not confirmed. Thus, the conclusion about strong regular shocks propagating in the stars' atmospheres may also be not true: the shocks are either weak $\left(V_{\mathrm{s}}<10-15 \mathrm{~km} \mathrm{~s}^{-1}\right)$ and/or appear in the stellar atmosphere rather seldom, not in every light cycle.

We observed the sample stars in the $\mathrm{H}_{2} \mathrm{O}$ line at $\lambda=1.35 \mathrm{~cm}$ on the RT-22 radio telescope of the Radio Astronomy Observatory (Astrospace Center of the Lebedev Institute of Physics, Russian Academy of Sciences) in Pushchino

\footnotetext{
${ }^{1}$ Sternberg Astronomical Institute, Moscow State University, 13 Universitetskij Prospekt, Moscow, 119899 Russia

${ }^{2}$ Instituto Nacional de Astrofísica, Óptica y Electrónica, Luis Enrique Erro No. 1, Apdo Postal 51 y 216, 72840 Tonantzintla, Puebla. México
} 
(Moscow Region). The studies of the correlation of the $\mathrm{H}_{2} \mathrm{O}$ maser emission with the light variability for various Mira Ceti type stars were undertaken earlier, see Berulis et al. (1998) and references therein. The basic result of our $\mathrm{H}_{2} \mathrm{O}$ observations was confirmation of the correlation between the variations of the visual brightness and $\mathrm{H}_{2} \mathrm{O}$ maser: in the stars we have studied the variations of the $\mathrm{H}_{2} \mathrm{O}$ maser follow the visual-brightness variability with some phase delay $\Delta \varphi$, from 0.1 to $0.4 P$. An example is the star U Ori, for which the most complete set of data has been obtained and which shows a variability of $\Delta \varphi$ with a superperiod of about 9 yr (Berulis, Lekht, \& Pashchenko 1994), implying a variable distance of the maser from the star.

So far, the most interesting result was obtained for the star $\mathrm{R}$ Leo. In $\mathrm{R}$ Leo, a strong flare in the $\mathrm{H} \alpha$ line happened in 1996 May; its duration was about one month. In 1997 December $\mathrm{R}$ Leo flared in the $\mathrm{H}_{2} \mathrm{O}$ line (Esipov et al. 1999). We connect these two flares with propagation of a shock wave in the inner layers of the circumstellar envelope, to distances of up to $\sim 10^{14} \mathrm{~cm}$ from the star center, where the masering $\mathrm{H}_{2} \mathrm{O}$ molecules are localized. In 1998-1999 we also observed similar sequences of the flares $\left(\mathrm{H} \alpha\right.$, then $\left.\mathrm{H}_{2} \mathrm{O}\right)$ in $\mathrm{R}$ Cas and U Aur; details will be published elsewhere.

To explain the correlation between he light curve and maser variations, Rudnitskij \& Chuprikov (1990) considered a mechanism of direct impact of the shock on the region of maser generation of the $\mathrm{H}_{2} \mathrm{O}$ molecules via modification of the power of the maser collisional pumping. Our results show that this mechanism may be true at least for $\mathrm{R} \mathrm{Leo,} \mathrm{R}$ Cas, and $\mathrm{U}$ Aur, where the two isolated-in-time events (the $\mathrm{H} \alpha$ flare and the $\mathrm{H}_{2} \mathrm{O}$ line flare) may be caused by the same shock propagating outward and consecutively initiating these two events, separated in time by almost two light cycles of the stars.

\section{References}

Berulis, I. I., Lekht, E. E., \& Pashchenko, M. I. 1994, Astron. Lett., 20, 115

Berulis, I. I., Lekht, E. E., Munitsyn, V. A., \& Rudnitskij, G. M. 1998, Astron. Reports, 42, 346

Esipov, V. F., Pashchenko, M. I., Rudnitskij, G. M., \& Fomin, S. V. 1999, Astron. Lett., 25, 672

Gillet, D., Maurice, E., \& Baade, D. 1983, A\&A, 128, 384

Joy, A. H. 1954, ApJS, 1, 39

Rudnitskij, G. M. \& Chuprikov, A. A. 1990, Soviet Ast., 34, 147 